\title{
Influencia del Calor Aportado y Metal de Aporte Sobre las Propiedades Mecánicas y la Microestructura de Juntas Soldadas por FCAW de Acero Microaleado de Alta Resistencia
}

\author{
César Marconi ${ }^{1}$, María José Castillo ${ }^{1,2}$, Leonardo Boccanera ${ }^{1}$, Mabel Ramini $^{1}$ \\ 1 Centro de Desarrollo y Tecnología de Materiales - DEYTEMA, Facultad Regional San Nicolás, Universidad Tecnológica Nacional, \\ Buenos Aires, Argentina. \\ 2 Escuela de Tecnología, Universidad Nacional del Noroeste de la Provincia de Buenos Aires - UNNOBA, Junín, Buenos Aires, \\ Argentina.
}

Recibido: 30 Mar., 2015

Aprobado: 21 Mayo, 2015

E-mail: cmarconi@frsn.utn.edu.ar (CM)
Resumen: Actualmente, los aceros microaleados son ampliamente utilizados en reemplazo de los aceros al C y C-Mn por sus ventajas en cuanto a propiedades mecánicas, resistencia a la corrosión y soldabilidad, permitiendo una reducción de peso de los vehículos y estructuras sin detrimento de su resistencia. Cuando estos aceros son soldados, el ciclo térmico de la soldadura provoca cambios microestructurales que modifican sus propiedades originales. El calor aportado (HI: heat input) es una de las principales variables a tener en cuenta cuando se evalúan estas modificaciones. Las propiedades finales de la unión soldada también se definen por el tipo de consumible utilizado. El objetivo de este trabajo es analizar las propiedades mecánicas y la microestructura de juntas soldadas a tope de un acero microaleado con dos grados de metal de aporte y diferentes HI. Como resultado del trabajo se observó un aumento considerable de tamaño de grano en la ZAC adyacente a la línea de fusión para todas las condiciones, siendo este efecto más marcado cuando se soldó con alto Hl; y un ablandamiento en la ZAC de grano fino. Prácticamente se mantuvieron los valores de resistencia a la tracción al soldar con ambos consumibles, con un mejor desempeño cuando se soldó con bajo HI. La tenacidad en la ZAC mejoró con la disminución del HI.

Palabras clave: Aceros microaleados; Propiedades mecânicas; Microestructura; Junta soldada; Alambres tubulares.

\section{Influence of Heat Input and Filler Metal on Mechanical Properties and Microstructure of Microalloyed High Strength Steel Welded by FCAW}

\begin{abstract}
Currently, microalloyed high strength steels are being widely used to replace $\mathrm{C}$ and C-Mn steels for their advantages in terms of mechanical properties, corrosion resistance and weldability, allowing weight reduction of vehicles and structures without impairing their strength. When these steels are welded, the welding heat cycle causes microstructural changes that alter their original properties. The heat input (HI: heat input) is one of the main variables to consider when these modifications are evaluated. The final properties of the welded joint are also defined by the type of consumable used. The aim of this paper is to analyze the mechanical properties and microstructure of a butt welded microalloyed steel with two filler metal grades and different HI. A considerable increase grain size in the heat affected coarse grain zone, especially for high $\mathrm{HI}$; and a softening in fine grain zone were observed. The values of tensile strength at both welding consumables remained basically unaltered, with better performance when welded with low HI. The toughness in the HAZ was improved when the HI decreased.
\end{abstract}

Key-words: Microalloyed steels; Mechanical properties; Microstructure; Welded joint; Cored wire.

\section{Introducción}

Los aceros microaleados de alta resistencia han reemplazado a los aceros al carbono tradicionales en aplicaciones de la industria automotriz y de la construcción por sus ventajas en cuanto a propiedades mecánicas, resistencia a la corrosión y soldabilidad. Estos aceros logran sus propiedades mecánicas, especialmente altos niveles de resistencia, mediante el agregado de microaleantes y procesos termomecánicamente controlados. Este proceso permite controlar la recristalización de la austenita, el crecimiento de 
Influencia del Calor Aportado y Metal de Aporte Sobre las Propiedades Mecánicas y la Microestructura de Juntas Soldadas por FCAW de Acero Microaleado de Alta Resistencia

grano y la transformación de austenita a ferrita mediante un enfriamiento programado y la adición de pequeñas cantidades de elementos microaleantes, tales como Ti, $\mathrm{Nb}$ y V. Se pueden definir entonces, los aceros microaleados como aquellos que contienen pequeñas cantidades, generalmente menores del 0,15\%, de elementos de aleación formadores de carburos, nitruros y carbonitruros los cuáles controlan directa o indirectamente la resistencia y otras propiedades de los aceros estructurales. De este modo se logra incrementar la resistencia por efecto del refinamiento de grano y el endurecimiento por precipitación [1].

Cuando estos aceros son soldados, el ciclo térmico de la soldadura causa alteraciones en la composición, distribución, tamaño y fracción volumétrica de los precipitados mediante la disolución, crecimiento y reprecipitación de los mismos. Estas transformaciones dan lugar a cambios microestructurales, tensiones residuales y modificaciones de resistencia y tenacidad a la fractura siendo la variable de mayor peso el HI [2]. Varios estudios de simulación han mostrado la influencia del $\mathrm{HI}$ sobre el tamaño de la zona afectada por el calor (ZAC) y el ablandamiento producido a medida que aumenta la distancia a la línea de fusión (LF) [3,4]. Sin embargo, dada la complejidad que se tiene al evaluar la microestructura y propiedades mecánicas y, posteriormente, relacionarlas con los ciclos térmicos para la química de cada acero, es importante verificar estas predicciones mediante métodos experimentales. Es, por lo tanto, necesario entender la influencia del proceso de soldadura y de la química de la aleación sobre el ablandamiento de la ZAC, en procura de encontrar una forma de minimizar este efecto.

Algunos productos de esta transformación pueden fragilizar la ZAC reduciendo sus propiedades mecánicas sobre todo cuando la estructura es sometida a cargas cíclicas. En estas zonas se suelen iniciar y propagar microgrietas que podrían llevar a un colapso de la estructura soldada en unos instantes [5].

Debido al ciclo térmico de la soldadura, en algunas regiones de la ZAC se alcanzan temperaturas que promueven la transformación de la estructura ferrítico/perlítica del metal base en austenita. Los elementos estabilizadores de austenita como carbono o manganeso pueden ser segregados en ciertas zonas en las que, durante el enfriamiento, precipiten constituyentes M-A. Estos constituyentes consisten en austenita retenida y austenita de alto carbono transformada en martensita. La presencia de los mismos es afectada por el $\mathrm{HI}$, que influye especialmente en el tiempo de enfriamiento desde 500 a $800{ }^{\circ} \mathrm{C}\left(\Delta \mathrm{t}_{8 / 5}\right)$. Además, en la zona cercana a la LF se encuentra el mayor tamaño de grano, lo que podría producir conjuntamente con la martensita una pérdida de tenacidad [5-7].

En la selección del consumible para realizar una junta soldada existen diferentes opciones que el especialista en soldadura debe analizar. Una teoría muy difundida es la que propone que el metal de soldadura debe tener una resistencia igual o mayor al metal base, de manera que cuando se ensaye la junta a la tracción la fractura ocurra fuera de la soldadura. En la actualidad se analizan otros conceptos y las opciones que se presentan a la hora de seleccionar un consumible a partir de sus propiedades mecánicas son tres: "matching": cuando la resistencia a la tracción del metal de aporte es igual a la del metal base; "undermatching": cuando es menor y "overmatching": cuando es mayor. Cuando el acero tiene una resistencia a la tracción del orden de los $350 \mathrm{MPa}$, el uso de consumibles de la familia E7XXX provee una situación de "overmatching", si la resistencia del metal base aumenta hasta el orden de los $400 \mathrm{MPa}$, este mismo consumible se utiliza como "matching". Con el advenimiento de aceros de alta resistencia y la permanente evolución de los mismos no siempre se dispone de consumibles de igual o mayor resistencia y la opción de usar un metal de aporte de menor resistencia que la del metal base se vuelve más atractiva [8].

En este trabajo se evalúa la influencia del HI y de la selección del consumible sobre las propiedades mecánicas y la microestructura de juntas soldadas a tope de un acero microaleado.

\section{Materiales y Métodos}

\subsection{Caracterización del metal base}

En este trabajo se utilizó un acero microaleado de alta resistencia de $6 \mathrm{~mm}$ de espesor como metal base de las juntas soldadas. Este material fue caracterizado mediante análisis químico, medición de tamaño de grano, ensayos de dureza, de tracción y de tenacidad.

Las Tablas 1 y 2 muestran la composición química, así como también el carbono equivalente (Ceq IIW) y las propiedades mecánicas del acero base utilizado. Como elementos endurecedores por precipitación se destacan el $\mathrm{Nb}$ y $\mathrm{Ti}$, la presencia de estos elementos permite disminuir el contenido de $\mathrm{C}$ para aumentar la soldabilidad y 
tenacidad, al mismo tiempo que permiten alcanzar elevados valores de resistencia [1]. En la Figura 1 se muestra la microestructura del acero base obtenida mediante microscopía óptica y electrónica de barrido (SEM). Se observa una estructura ferrítica con algo de perlita $(P)$ y tamaño de grano uniforme de 5,4 $\mu$ m de diámetro en todo el espesor de la chapa.

Tabla 1. Composición química del metal base.

\begin{tabular}{ccccccccc}
\hline $\mathbf{C}$ & $\mathbf{M n}$ & $\mathbf{S i}$ & $\mathbf{S}$ & $\mathbf{P}$ & $\mathbf{C r}$ & $\mathbf{N i}$ & $\mathbf{M o}$ \\
0,082 & 1,223 & 0,132 & 0,006 & 0,019 & 0,029 & 0,028 & 0,010 \\
\hline $\mathbf{V}$ & $\mathbf{N b}$ & $\mathbf{A l}$ & $\mathbf{T i}$ & $\mathbf{C u}$ & $\mathbf{N}(\mathbf{p p m})$ & $\mathbf{B}$ (ppm) & $\mathbf{C e q}$ \\
0,003 & 0,043 & 0,058 & 0,037 & 0,04 & 40 & 2 & 0,32 \\
\hline
\end{tabular}

Tabla 2. Propiedades mecánicas del MB.

\begin{tabular}{ccccccc}
\hline $\mathbf{R}(\mathrm{MPa})$ & $\mathbf{R f}(\mathrm{Mpa})$ & $\mathbf{A}(\%)$ & $\begin{array}{c}\text { HV1 } \\
\text { (promedio) }\end{array}$ & $\mathbf{2 0 ^ { \circ } \mathrm { C }}$ & $\mathbf{- 2 0 ^ { \circ } \mathbf { C }}$ & $\mathbf{- 6 0 ^ { \circ } \mathbf { C }}$ \\
\hline 610 & 516 & 27 & 197 & 170 & 164 & 126 \\
\hline
\end{tabular}

R: resistencia a la tracción. Rf: tensión de fluencia. A: alargamiento. HV1: microdureza (1000 g).

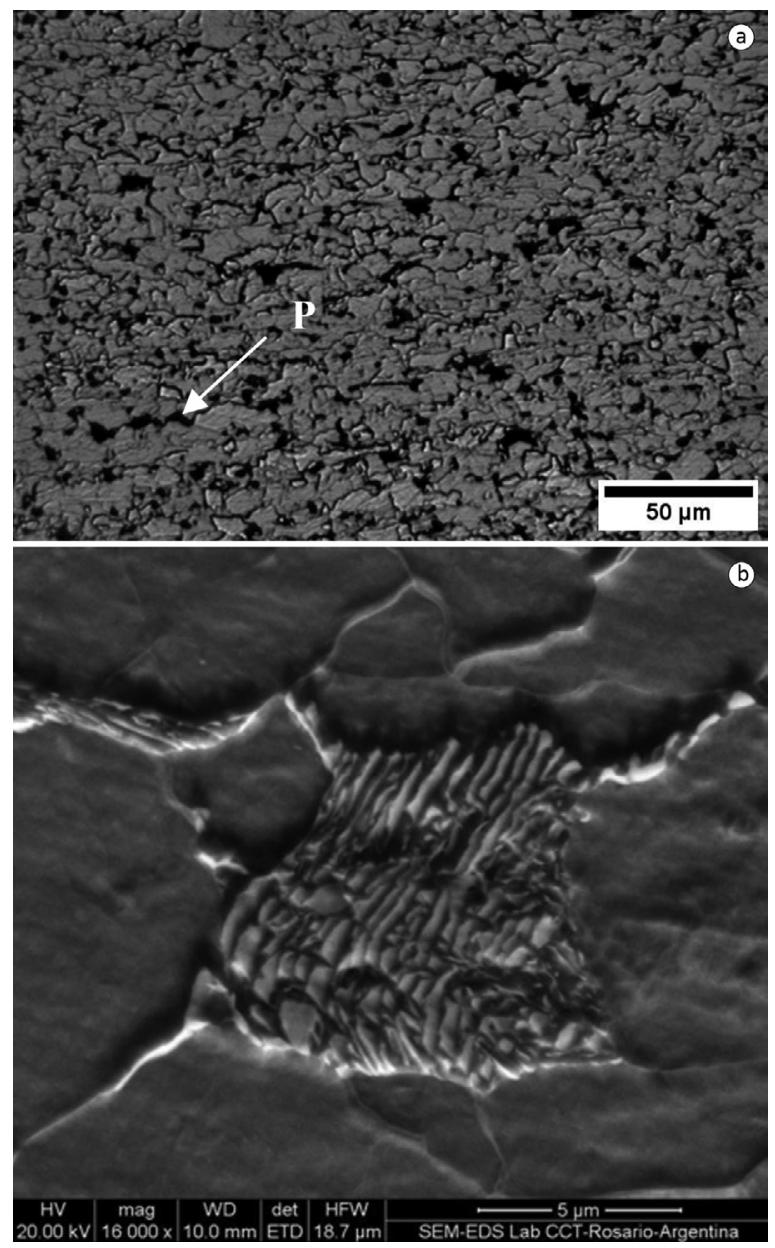

Figura 1. Microestructura del acero base, (a) microscopia óptica y (b) electrónica de barrido. 


\subsection{Soldadura de cupones de prueba}

Para la soldadura de las probetas se realizaron cupones de junta soldada a tope biselados en $V$ de $30^{\circ}$, según la norma AWS D1.1/D1.1M: 2010 [9]. El diseño de junta utilizado puede ser observado en la Figura 2.

El proceso de soldadura empleado fue FCAW-G. El material de aporte utilizado fue del tipo tubular con dos grados de resistencia, clasificados según norma como E71T1C (U) y E81T1Ni1C (M) [10,11]. La Tabla 3 muestra la composición química de metal de aporte puro para los consumibles $\mathrm{U}$ y $\mathrm{M}$ utilizados.

Como protección gaseosa se empleó la mezcla activa $\mathrm{Ar}-20 \% \mathrm{CO}_{2}$. Se obtuvieron dos condiciones de $\mathrm{HI}$ mediante la modificación del número de pasadas, la condición de alto HI fue obtenida por realización de cordones de soldadura de una pasada (1) y la condición de bajo HI fue obtenida mediante la realización de cordones de soldadura de tres pasadas (3).

La identificación de cada cupón de prueba, los parámetros de soldadura utilizados y el HI resultante se muestran en la Tabla 4.

\subsection{Análisis macro y microestructural}

Se realizaron cortes transversales al cordón soldado y se analizó la macro y microestructura. Todas las muestras se desbastaron con papeles abrasivos de carburo de silicio hasta grado 600, el pulido final se realizó utilizando pasta diamantada de 6 y $1 \mu \mathrm{m}$. La microestructura de soldadura se reveló con Nital $2 \%$ y se evaluó por microscopía óptica y electrónica de barrido.

Se definieron las diferentes zonas de la junta soldada: metal de soldadura (MS), zona afectada por el calor de grano grueso (ZAC GG), zona afectada por el calor de grano fino (ZAC GF) y metal base (MB).

Se clasificaron los diferentes constituyentes del MS, mediante el sistema propuesto por el Instituto Internacional de Soldadura (IIW) [12].

Se realizaron mediciones de tamaño de grano a través del método de las intersecciones, según la norma ASTM E 112-96 [13], en la adyacencia de la línea de fusión (LF), dentro de la ZAC GG y en la ZAC GF. En la ZAC GG se cuantificó el porcentaje de partículas M-A presentes en la estructura mediante la norma ASTM E 562-02 [14] a partir de microfotografías obtenidas mediante SEM.

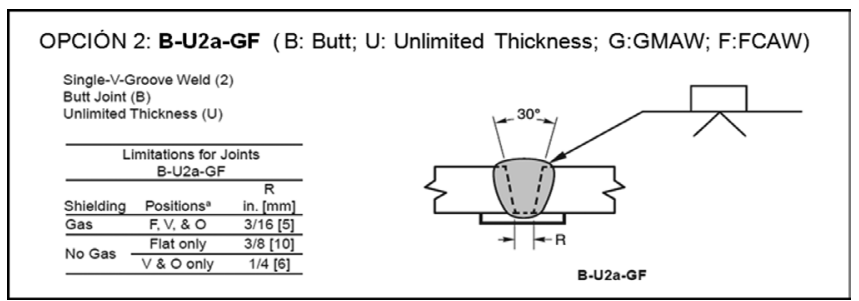

Figura 2. Diseño de junta utilizado.

Tabla 3. Composición química de metal de aporte puro.

\begin{tabular}{lccccccccc}
\hline Metal de Aporte & $\mathbf{C}$ & Si & Mn & S & P & V & Cr & Ni & Mo \\
E71T1C (U) & 0,044 & 0,53 & 1,29 & 0,007 & 0,011 & & & & \\
E81T1Ni1C (M) & 0,031 & 0,47 & 1,10 & 0,005 & 0,026 & 0,023 & 0,04 & 0,84 & 0,013 \\
\hline
\end{tabular}

Tabla 4. Parámetros de soldadura e identificación de los cupones.

\begin{tabular}{cccccc}
\hline Probeta & $\mathbf{N}^{\circ}$ de Pasadas & Corriente (A) & Tensión $(\mathbf{V})$ & $\begin{array}{c}\text { Vel. de Soldadura } \\
(\mathbf{m m} / \mathbf{s})\end{array}$ & $\begin{array}{c}\text { Calor aportado } \\
\mathbf{( k J / m m})\end{array}$ \\
$\mathbf{1 U}$ & 1 & 256 & 29,2 & 3,5 & 2,12 \\
$\mathbf{3 U}$ & 3 & 170 & 25,2 & 4,6 & 0,95 \\
$\mathbf{1 M}$ & 1 & 260 & 30,0 & 3,1 & 2,48 \\
$\mathbf{3 M}$ & 3 & 170 & 25,2 & 4,7 & 0,93 \\
\hline
\end{tabular}




\subsection{Ensayos mecánicos}

Se realizaron mediciones de microdureza mediante barridos desde el MS hasta el MB con 1000 gf de carga, las improntas fueron realizadas cada $0,5 \mathrm{~mm}$. Se efectuaron dos barridos por muestra, el primero en la región superior del cordón de soldadura y el segundo en la raíz del mismo. Los resultados obtenidos fueron promediados para cada zona de la junta soldada. El esquema de la Figura 3 muestra los barridos realizados.

Para el ensayo de tracción se maquinaron 2 probetas transversales de cada cupón de prueba según el esquema de la Figura 4. Las mismas fueron ensayadas siguiendo la norma ASTM E 8-04 [15].

Se realizaron pruebas de impacto Charpy- $\mathrm{V}$ a 20, -20 y $-60{ }^{\circ} \mathrm{C}$ según la norma ASTM E 23-02 [16], sobre probetas subsize mecanizadas con la entalla ubicada a $1 \mathrm{~mm}$ de la línea de fusión, abarcando las zonas de ZAC GG y GF como se muestra en el esquema de la Figura 5.

\section{Resultados y Discusión}

\subsection{Análisis macro y microestructural}

La Figura 6 muestra, a modo de ejemplo, una macrografía de las probetas soldadas con 1 pasada de soldadura (a) y otra realizada con 3 pasadas (b). En las mismas se aprecian las distintas zonas de una junta soldada. El estudio macroestructural reveló una disminución del ancho de la ZAC al soldar con bajo $\mathrm{HI}$, independientemente del grado de consumible utilizado [3]. Esto puede ser observado en la Figura 7 referente al análisis de los perfiles de microdureza.

En la Figura 8 se muestra la microestructura correspondiente al MS para cada una de las condiciones estudiadas. En las probetas soldadas con el consumible U se observa una estructura de ferrita primaria en borde de grano $(P F(G))$, ferrita con segundas fases alineadas (FS(A)) y algo de ferrita acicular (AF). Este último constituyente se observa en mayor proporción en las probetas soldadas con el consumible M junto con ferrita con segundas fases no alineadas (FS(NA)). Al comparar las composiciones químicas de los aportes utilizados (ver Tabla 3) se observa un importante contenido de $\mathrm{Ni}$ en el consumible $\mathrm{M}$, este elemento disminuye la temperatura de transformación del acero favoreciendo la formación de estructuras aciculares $[17,18]$.

En las Figuras 9 y 10 se observan microfotografías obtenidas por microscopía óptica correspondientes a la ZAC GG y GF de las probetas soldadas. En ambas zonas se observa un refinamiento de grano en las probetas soldadas con 3 pasadas.

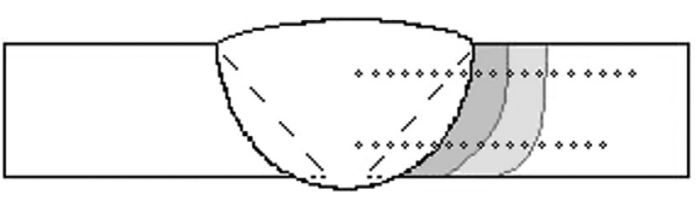

Figura 3. Barridos de microdureza realizados en las probetas de análisis.

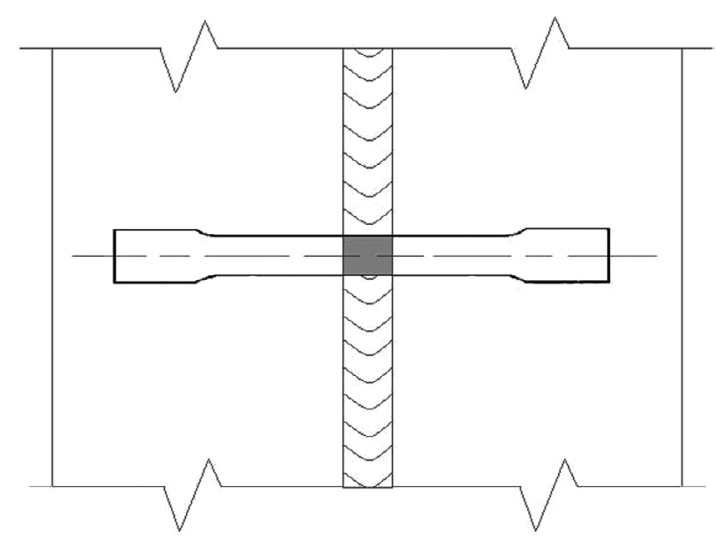

Figura 4. Esquema del cupón de soldadura con la ubicación de la probeta de tracción.

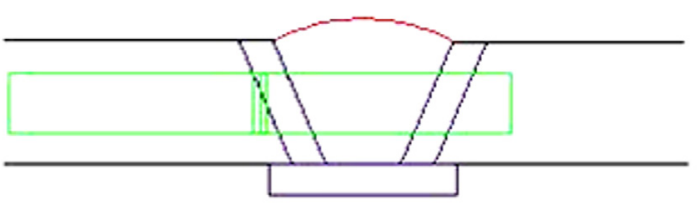

Figura 5. Esquema de la junta soldada con la ubicación de las probetas de Charpy-V subsize.

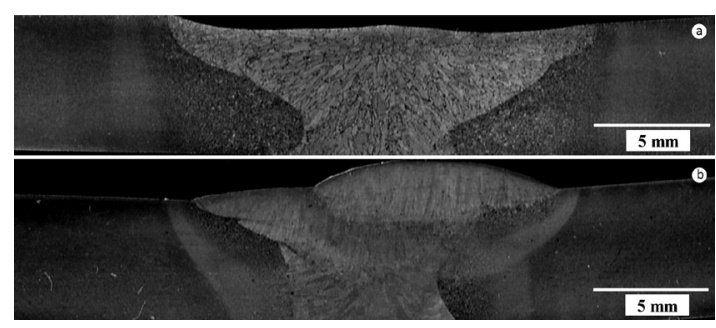

Figura 6. Macrografía de una probeta soldada con 1 pasada (a) y otra con 3 pasadas (b). 
Influencia del Calor Aportado y Metal de Aporte Sobre las Propiedades Mecánicas y la Microestructura de Juntas Soldadas por FCAW de Acero Microaleado de Alta Resistencia
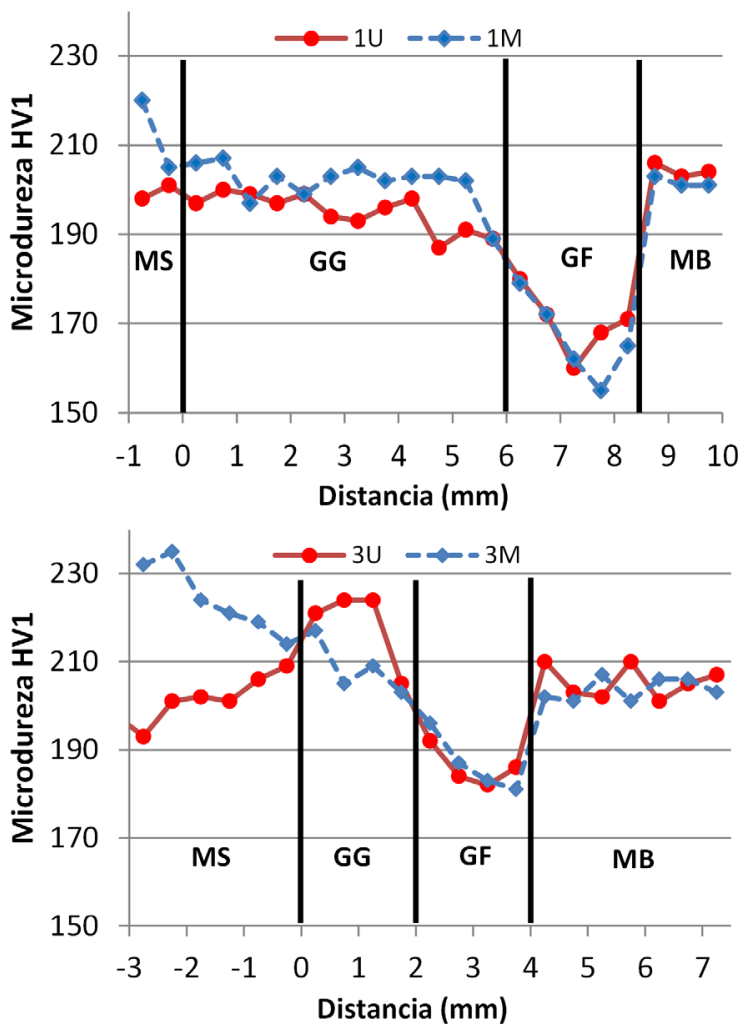

Figura 7. Perfiles inferiores de microdureza para alto y bajo HI respectivamente.
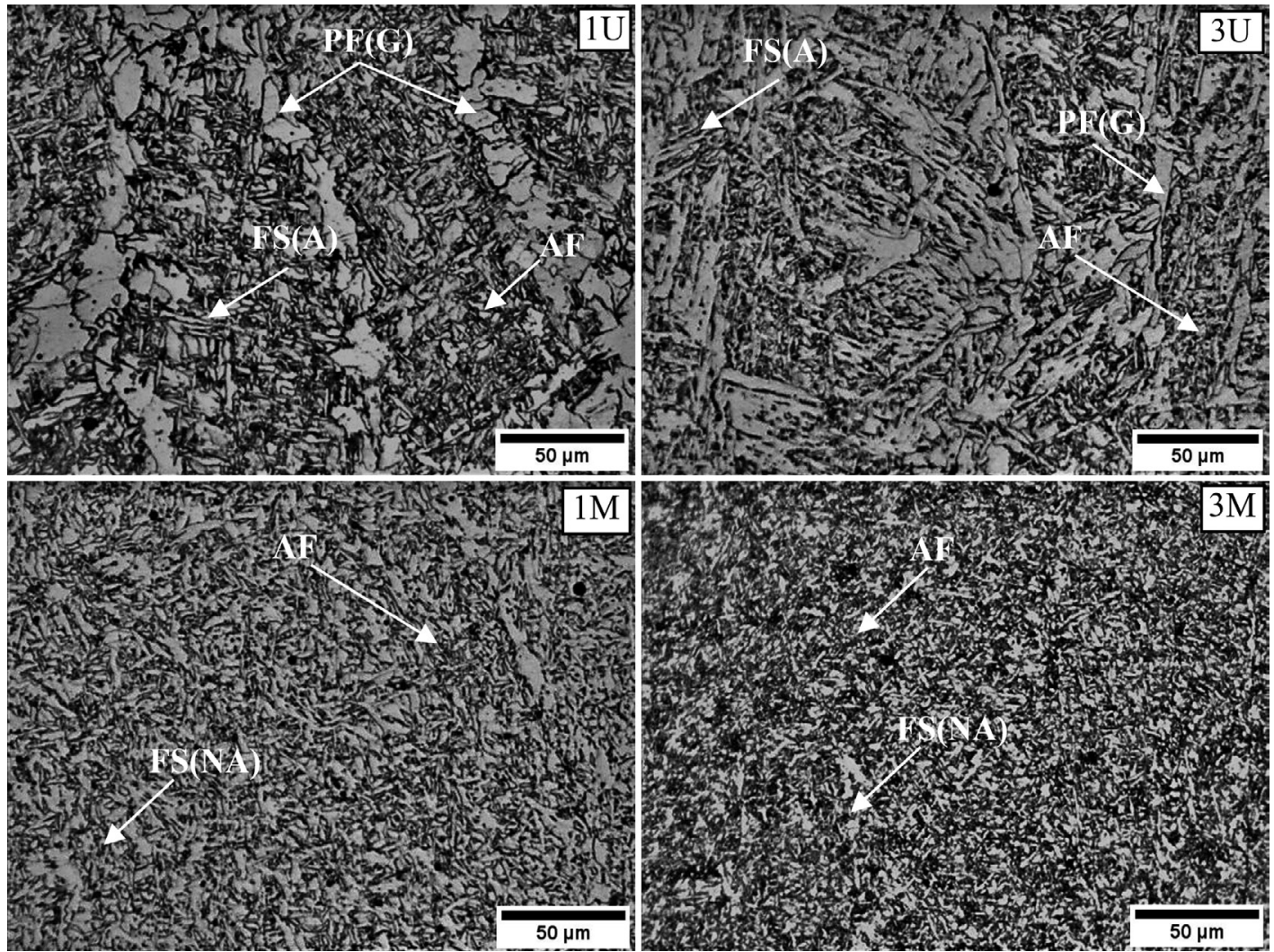

Figura 8. Microestructura del metal de soldadura. 

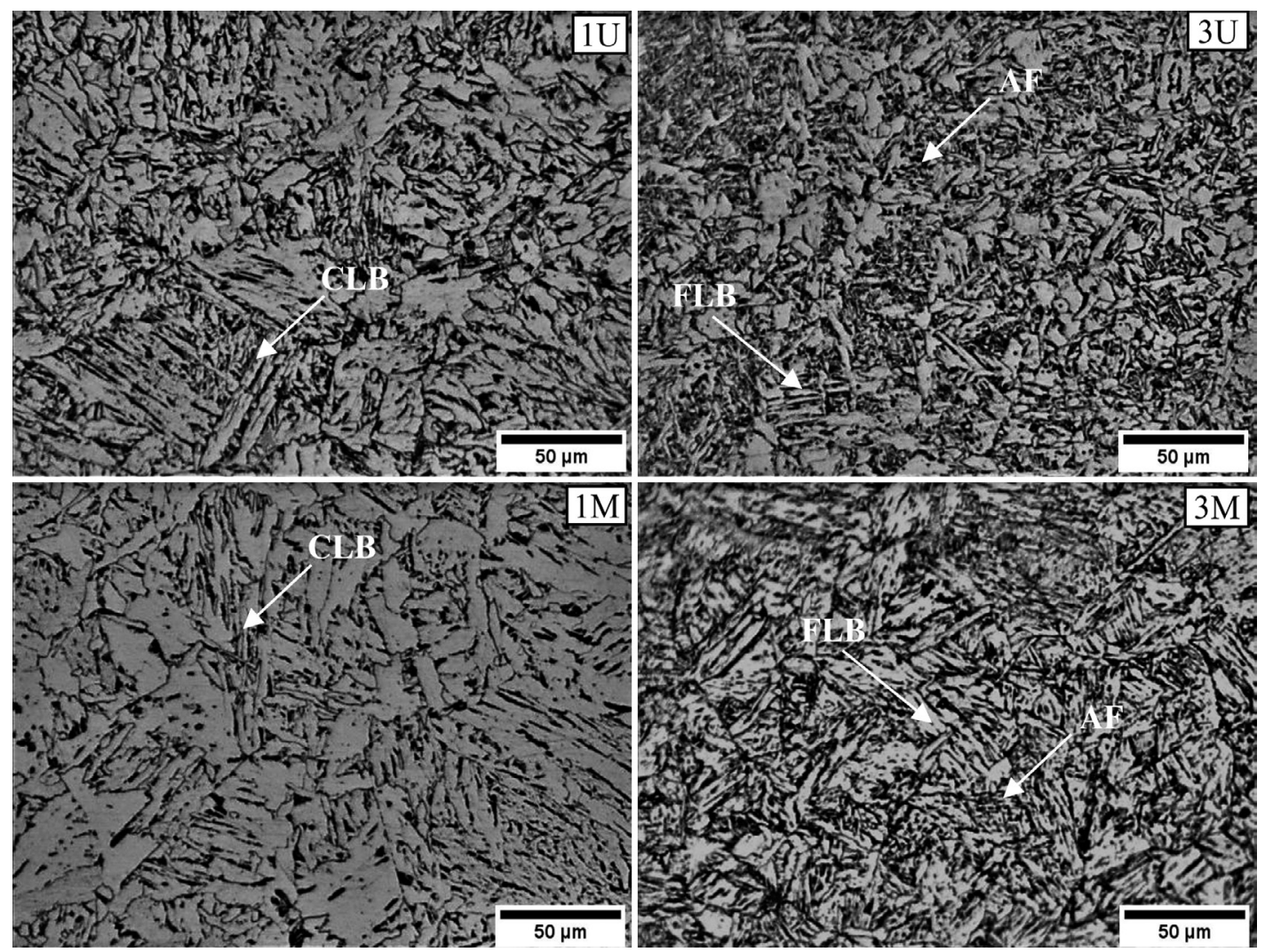

Figura 9. Microestructura de la ZAC GG de las diferentes probetas de estudio.
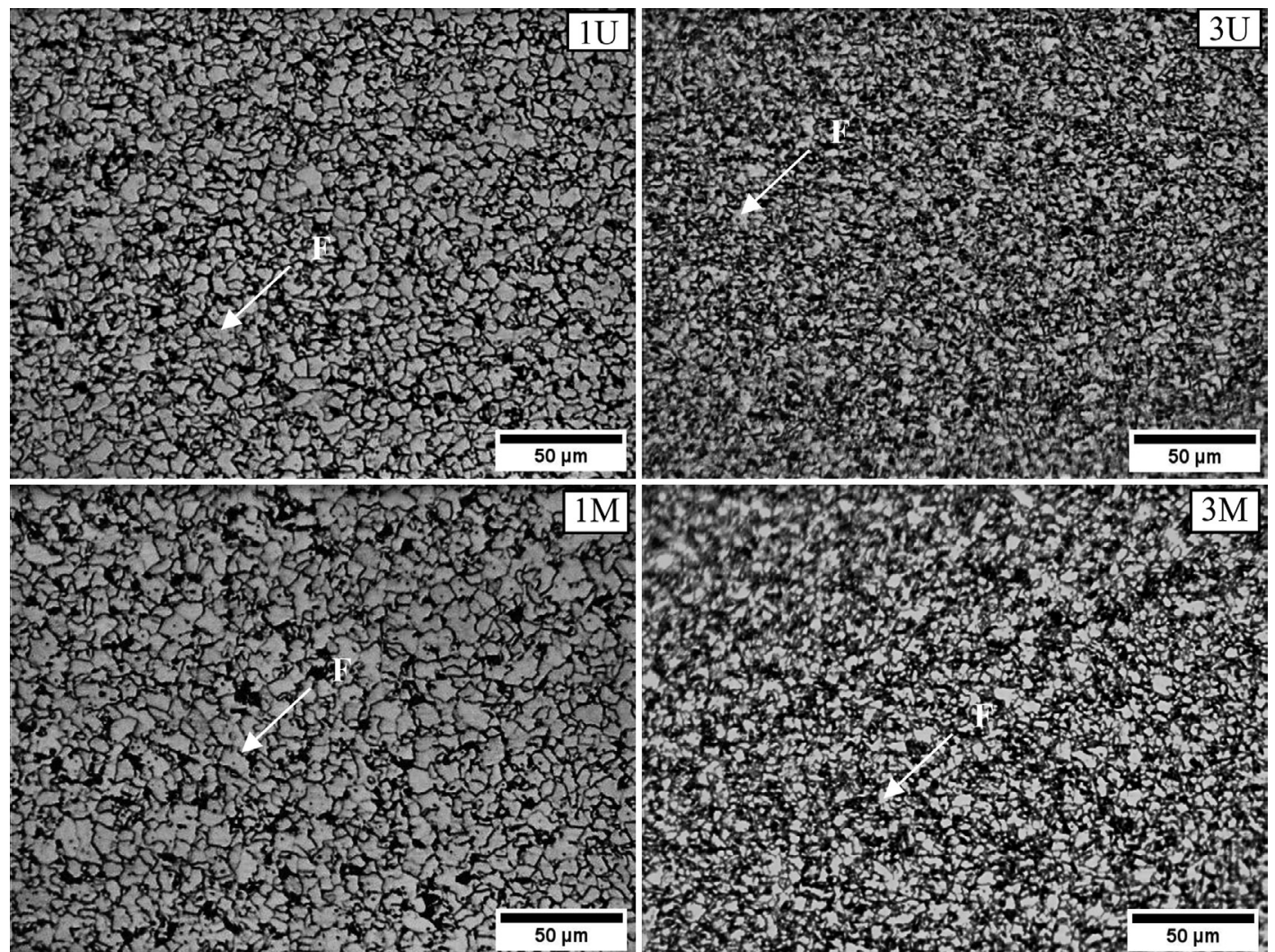

Figura 10. Microestructura de la ZAC GF de las diferentes probetas de estudio. 
Influencia del Calor Aportado y Metal de Aporte Sobre las Propiedades Mecánicas y la Microestructura de Juntas Soldadas por FCAW de Acero Microaleado de Alta Resistencia

En cuanto a la microestructura de la ZAC GG se observa para las probetas con alto $\mathrm{HI}$ una estructura de bloques de ferrita con listones de bainita gruesa (CLB: coarse lath bainite). Para las probetas de bajo $\mathrm{HI}$ se destaca un aumento de ferrita acicular (AF) a expensas de un menor porcentaje de bloques de ferrita y finos listones de bainita (FLB: fine lath bainite). En la ZA C GF se observa una estructura de ferrita poligonal recristalizada $(F)[4,19]$.

Los resultados de la medición de tamaño de grano en la ZAC GG y ZAC GF se muestran en la Tabla 5.

Comparando los tamaños de grano de la ZAC GF se observa un refinamiento de la estructura cuando se suelda con tres pasadas, la mayor velocidad de enfriamiento asociada a un menor HI podría determinar este comportamiento, el cual también es evidenciado en las micrografías de la Figura 10 [18].

En las probetas soldadas con una sola pasada se observa un aumento considerable del tamaño de grano de la ZAC GG en la zona aledaña a la LF. En esta zona es de esperar una completa disolución de los carburos por lo cual, cuando se suelda con alto $\mathrm{HI}$, el mayor tiempo de permanencia a temperaturas de austenización y la ausencia de precipitados que anclen el borde de grano resulta en un tamaño de grano grosero $[3,20]$.

La Tabla 6 permite observar los resultados obtenidos de la cuantificación de partículas M-A.

La Figura 11 muestra las microfotografías obtenidas mediante SEM donde se observan las partículas M-A.

Según puede observarse en la Tabla 6, el porcentaje de constituyente M-A aumenta con el HI, la bibliografía consultada reporta que para aceros de alta resistencia la cantidad de constituyente $\mathrm{M}$-A se incrementa rápidamente cuando $\Delta \mathrm{t}_{8 / 5}$ aumenta por encima de los $10-20 \mathrm{~s}$, alcanzando un máximo y luego disminuyendo debido a la descomposición de carburos en ferrita [20,21].

\subsection{Ensayos mecánicos}

\subsubsection{Microdureza}

La Figura 7 muestra los perfiles inferiores de microdureza de las probetas soldadas con los consumibles $U$ y $M$, para las condiciones de alto y bajo $\mathrm{HI}$ respectivamente. El mismo comportamiento fue evidenciado en los perfiles superiores. Aunque como se puede observar en la Figura 6, el ancho correspondiente a cada zona varía. Por ello, a los efectos de poder comparar los resultados obtenidos en cada región, se optó por graficar solo los perfiles inferiores.

En la Figura 12 se muestran los valores promedio (perfil superior e inferior) de microdureza correspondientes al MS.
Tabla 5. Tamaño de grano en las diferentes zonas de la junta soldada.

\begin{tabular}{cccc}
\hline \multicolumn{3}{c}{ Tamaño de grano $(\boldsymbol{\mu m})$} \\
Probeta & Metal base & ZAC GF & ZAC GG * \\
1U & 5,4 & 5,8 & 148 \\
3U & & 4,3 & 48 \\
1M & & 5,9 & 145 \\
3M & & 4,1 & 60 \\
\hline
\end{tabular}

*Adyacente a la línea de fusión.

Tabla 6. Resultados de la cuantificación de partículas M-A.

\begin{tabular}{cccc}
\hline Probeta & $\mathbf{H I}(\mathbf{k J} / \mathbf{m m})$ & $\% \mathbf{M}-\mathbf{A}$ & $\boldsymbol{\Delta t}_{\mathbf{8} / \mathbf{5}}$ \\
$\mathbf{1 U}$ & 2,12 & 5 & $28 \mathrm{~s}$ \\
$\mathbf{3 U}$ & 0,95 & 3,2 & $11 \mathrm{~s}$ \\
$\mathbf{1 M}$ & 2,48 & 5,1 & $33 \mathrm{~s}$ \\
$\mathbf{3 M}$ & 0,93 & 2,3 & $11 \mathrm{~s}$ \\
\hline
\end{tabular}

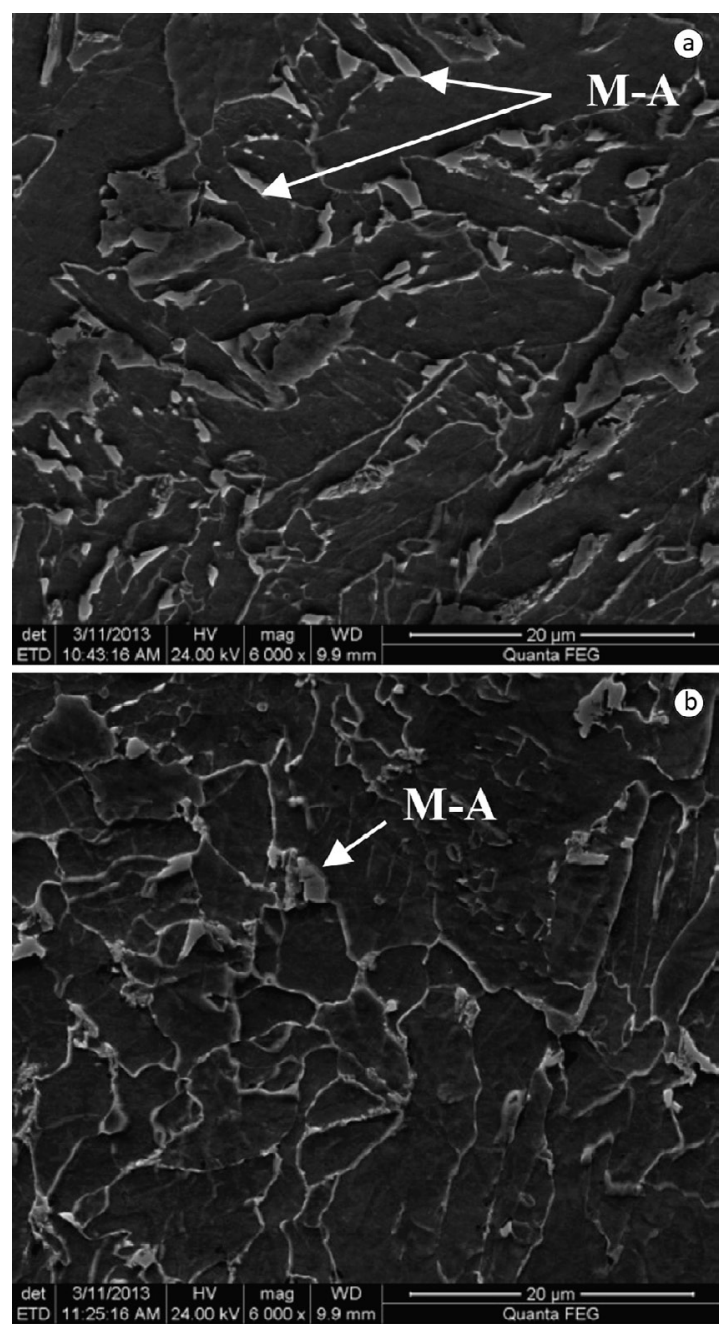

Figura 11. Partículas M-A cuantificadas (a) Probeta $1 \mathrm{M}$ y (b) Probeta 3M. 
Los resultados obtenidos muestran un endurecimiento de la estructura del MS cuando se suelda con consumibles de resistencia similar al MB (probetas $\mathrm{M}$ ). No se observa un efecto trascendente del $\mathrm{HI}$ sobre la microdureza, este pudo haber sido enmascarado por los cambios realizados en el número de pasadas. Si bien es de esperar un aumento de dureza al soldar con bajo $\mathrm{HI}$, el recalentamiento provocado por pasadas subsiguientes, para las configuraciones de bajo HI habría compensado el efecto directo de la variación del HI sobre la microestructura.

En la Figura 13 se muestran los promedios de los valores de ambos barridos de microdureza (superior e inferior, Figura 3), tomados para las diferentes zonas: ZAC GG y ZAC GF. Tanto en la ZAC GG como en la ZAC GF se observa un ablandamiento del MB más pronunciado en la ZAC GF correspondiente a una estructura de ferrita poligonal recristalizada [22]. Este ablandamiento se hace más pronunciado cuando se suelda con 1 pasada debido a que en estas condiciones el tamaño de grano se ha mantenido muy similar al del MB (Tabla 5).

Con el consumible $U$ cuando se suelda con una sola pasada se observa un ablandamiento mayor que con el consumible M en la ZAC GG.

\subsubsection{Ensayo de tracción}

En la Tabla 7 se presentan los resultados del ensayo de tracción para las juntas soldadas en diferentes condiciones. Además, se indican los valores de resistencia obtenida para el MB, como así también para los metales de aporte empleados. Por otro lado, en la última columna de la tabla se indica la región de la junta donde ocurrió la fractura.

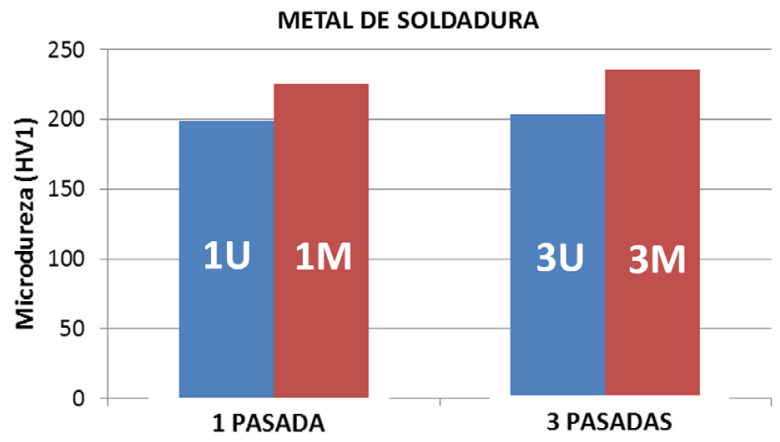

Figura 12. Microdureza del metal de soldadura.

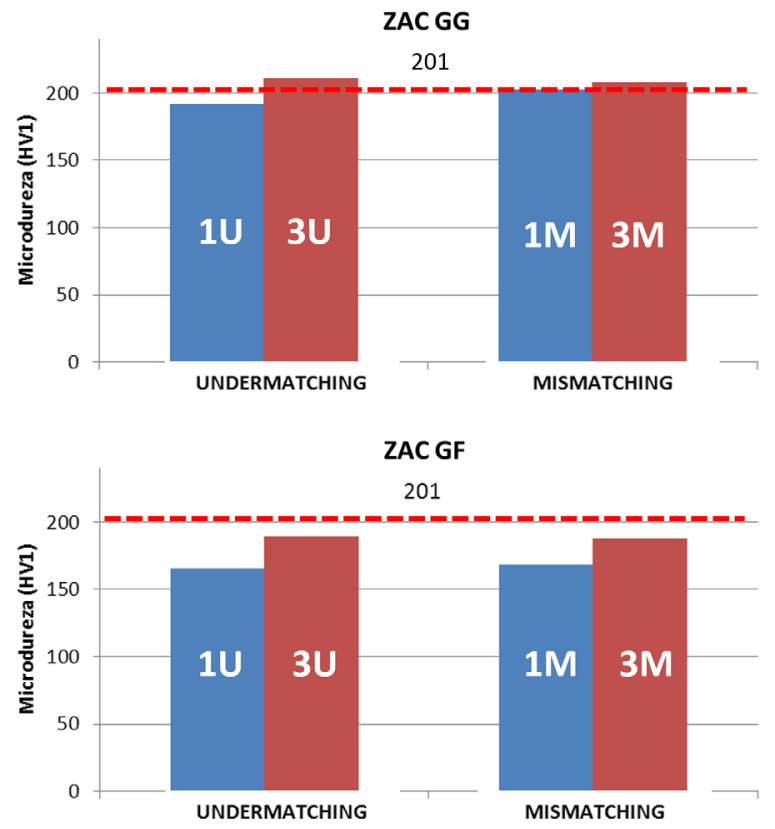

Figura 13. Microdureza promedio de las diferentes zonas de la junta soldada. 
Prácticamente se mantienen los valores de resistencia a la tracción al soldar con ambos consumibles (U y M), con un mejor comportamiento cuando se suelda con 3 pasadas. En general se observó una tendencia a la disminución de la resistencia con el aumento del HI. Este resultado puede ser atribuido a la variación del tamaño de grano en la ZAC y a la evolución de la cinética de disolución - reprecipitación de carbonitruros [6].

El aumento del HI provocó una pérdida de resistencia comparable para ambos consumibles (U y M).

En todos los casos se observa una significativa pérdida de alargamiento y de tensión de fluencia de aproximadamente $53 \%$ y $13 \%$ respecto del consumible, respectivamente. Este efecto puede ser atribuido a un comportamiento típico de probetas de tracción de materiales compuestos con diferentes tensiones de fluencia [23].

La Figura 14 muestra las macrofotografías de la zona de fractura de cada una de las probetas ensayadas. Al comparar las zonas de fractura de las probetas soldadas con el consumible $U$, se observa que la muestra $1 U$ falla en la ZAC GG, mientras que en la $3 \mathrm{U}$ la fractura ocurre en el MS. En la probeta $1 \mathrm{U}$ este comportamiento se corresponde con la presencia de un tamaño de grano basto y baja dureza en la región de la fractura. En cambio, en la probeta $3 \mathrm{U}$ el comportamiento es opuesto, la dureza es mayor a la obtenida en el MS y el tamaño de grano inferior al de la probeta $1 \mathrm{U}$.

Este análisis indica que los diferentes cambios microestructurales observados en la ZAC GG para cada una de las condiciones de $\mathrm{HI}$ se correlacionan con los resultados obtenidos. El excesivo engrosamiento del grano austenítico en la ZAC GG resulta generalmente en detrimento de las propiedades mecánicas de dicha zona [17].

Cuando se suelda con el consumible $M$ la fractura se ubica en la ZAC GF, siendo ésta la zona de la ZAC de menor dureza. El hecho de que la fractura no ocurra en la ZAC GG podría deberse a la difusión de elementos de aleación desde el MS hacia dicha zona, aumentando su resistencia por solución sólida y a la presencia de estructuras martensíticas y bainíticas de mayor dureza [4].

Tabla 7. Resultados del ensayo de tracción.

\begin{tabular}{|c|c|c|c|c|c|c|c|c|c|c|}
\hline \multirow{2}{*}{ Cupón } & \multicolumn{3}{|c|}{ Metal Base } & \multicolumn{3}{|c|}{ Consumible } & \multicolumn{3}{|c|}{ Junta Soldada } & \multirow{2}{*}{$\begin{array}{l}\text { Zona de } \\
\text { Fractura }\end{array}$} \\
\hline & $\mathrm{Rf}(\mathrm{MPa})$ & $\mathrm{R}(\mathrm{MPa})$ & A (\%) & $\mathrm{Rf}(\mathrm{MPa})$ & $\mathrm{R}$ (MPa) & A (\%) & Rf $\mathrm{MPa}$ ) & $\mathrm{R}$ (MPa) & A (\%) & \\
\hline $1 \mathrm{U}$ & \multirow{4}{*}{516} & \multirow{4}{*}{610} & \multirow{4}{*}{27} & \multirow{2}{*}{550} & \multirow{2}{*}{590} & \multirow{2}{*}{32} & 479 & 587 & 17 & ZAC-GG \\
\hline $3 \mathrm{U}$ & & & & & & & 490 & 605 & 20 & MS \\
\hline $1 \mathrm{M}$ & & & & \multirow{2}{*}{570} & \multirow{2}{*}{610} & \multirow{2}{*}{30} & 494 & 600 & 14 & ZAC-GF \\
\hline $3 \mathbf{M}$ & & & & & & & 511 & 627 & 20 & MB \\
\hline
\end{tabular}

Rf: tensión de fluencia. R: resistencia a la tracción. A: alargamiento.

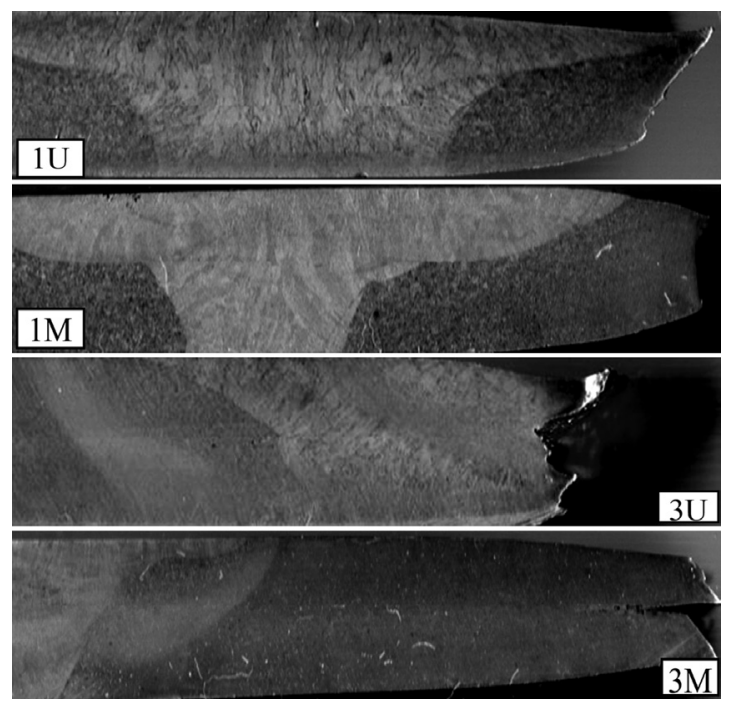

Figura 14. Macrofotografías de la zona de fractura de las probetas de tracción. 

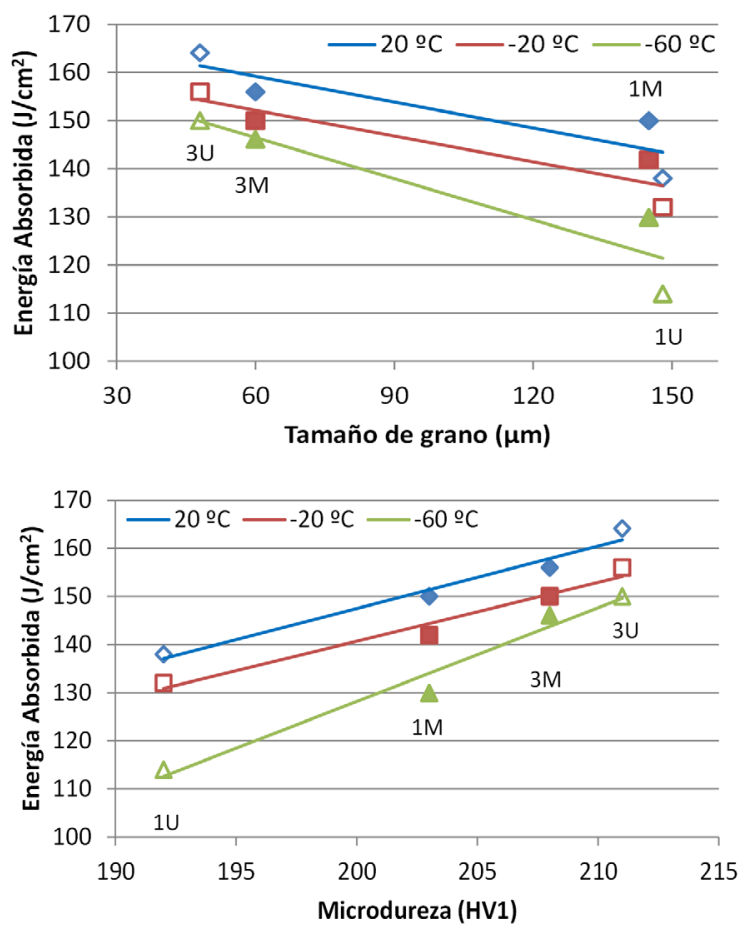

Figura 15. Tenacidad en función del tamaño de grano y la microdureza.

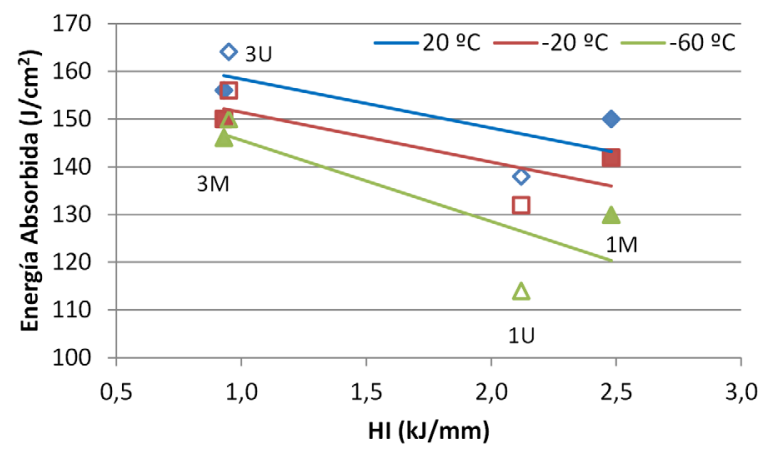

Figura 16. Tenacidad en función del calor aportado.

\subsubsection{Ensayo de tenacidad}

En cuanto a los resultados de las pruebas de impacto, según los gráficos de la Figura 15, se observó un aumento de tenacidad al disminuir el tamaño de grano y aumentar la dureza de la ZAC GG. Los menores valores de tenacidad se obtuvieron para la condición de alto HI como se puede observar en el gráfico de la Figura 16 [6,7,21]. Un menor tamaño de grano, estructuras ferrítco/bainíticas más finas y menor porcentaje de M-A justifican este comportamiento [24].

Al comparar los resultados obtenidos para cada consumible, se observó que para bajo $\mathrm{HI}$ las probetas soldadas con $\mathrm{U}$ presentaron mayor tenacidad que las soldadas $\operatorname{con} \mathrm{M}$, mientras que para alto $\mathrm{HI}$ la pérdida de tenacidad es mayor para el consumible $U$ asociado a un mayor ablandamiento.

\section{Conclusiones}

En las uniones soldadas se observó un aumento considerable de tamaño de grano en la ZAC GG, siendo este efecto más marcado cuando se soldó con una sola pasada o alto HI. 
Influencia del Calor Aportado y Metal de Aporte Sobre las Propiedades Mecánicas y la Microestructura de Juntas Soldadas por FCAW de Acero Microaleado de Alta Resistencia

El ancho de la ZAC disminuyó considerablemente cuándo se soldó con 3 pasadas o bajo HI.

Los resultados obtenidos mostraron mayor dureza en el MS cuando se soldó con el consumible $\mathrm{M}$ y no se observó un efecto significativo del HI en esta zona. Tanto en la ZAC GG como en la ZAC GF se observó un ablandamiento del MB más pronunciado en la ZAC GF y cuando se soldó con 1 pasada o alto HI.

Prácticamente se mantuvieron los valores de resistencia a la tracción al soldar con ambos consumibles ( $U$ y M), con un mejor comportamiento cuando se soldó con 3 pasadas o bajo HI.

Para cada consumible la tenacidad mejoró cuando disminuyó el HI relacionado al menor tamaño de grano y mayor dureza de la ZAC GG. Con el aumento del HI cayeron los valores de tenacidad, siendo más marcado este efecto cuando se soldó con el consumible $U$.

\section{Referencias}

[1] Illescas S. Estudio del efecto de los elementos microaleantes en un acero HSLA [tesis de maestría]. Cataluña: Universidad Politécnica de Cataluña. Departamento de Ciencia de los Materiales e Ingeniería Metalúrgica; 2008.

[2] Gonzalez Palma R. Estudio de la tenacidad a la fractura relacionada con la microestructura en la zona afectada por el calor (ZAC) de los aceros microaleados para plataformas Off-Shore [tesis doctoral]. Cádiz: Facultad de Ciencias, Universidad de Cádiz; 1994.

[3] Shome M, Effect of heat input on austenite grain size in the heat effected zone of HSLA-100 steel. Journal of Materials Science \& Engineering. 2006;445-446:454-460,.

[4] Shukla R, Das SK, Ravi Kumar B, Ghosh SK, Kundu S, Chatterjee S. An ultra-low carbon, thermomechanically controlled processed microalloyed steel: microstructure an mechanical properties. Metallurgical and Materials Transactions. A, Physical Metallurgy and Materials Science. 2012;43(12):4835-4845. http://dx.doi. org/10.1007/s11661-012-1273-7.

[5] González-Palma R, Muñoz A, Caamaño FJ, Portela JM. Ciclo térmico de aceros microaleados de alta resistencia $450 \mathrm{EMZ}$ tipo II en el simulador de soldadura. In Asociación Española de Ingeniería Mecánica. XVIII Congreso Nacional de Ingeniería Mecánica; 2010 Novembre 3, 4, y 5; Ciudad Real. Ciudad Real: Universidad de Castilla-La Mancha; 2010.

[6] LiY, Crowther DN, Green MJW, Mitchell PS, Baker TN. The effect of vanadium and niobium on the properties and microstructure of the intercritically reheated coarse grained heat affected zone in low carbon. ISIJ International. 2001;41(1):46-55. http:// dx.doi.org/10.2355/isijinternational.41.46.

[7] Ikawa H., Oshige H., Tanoue T. Effect of martensite-austenite constituent on haz toughness of a high strength steel. Quebec: IIW; 1980. (International Institute of Welding Document, n. IX-1156-80).

[8] Miller D. K. Use undermatching weld metal where advantageous, Welding Innovation. 1997; XIV(1).

[9] An American National Standard Institute - ANSI. AWS D1.1/ D1.1M:2010: Structural welding code-steel. Miami: American Welding Society; 2010.

[10] American Welding Society - AWS. AWS A5.20/A5.20M:2005: Specification for carbon steel electrodes for flux cored arc welding. Danvers: AWS; 2005.

[11] American Welding Society - AWS. AWS A5.29/A5.29M:2010: Specification for low-alloy steel electrodes for flux cored arc welding. Danvers: AWS; 2010.

[12] Guide to the Light Microscope Examination of Ferrite Steel Weld Metals. Quebec: IIW; 1988. (International Institute of Welding Document, n. IX-1533-88).

[13] American Standard for Testing Materals - ASTM. ASTM E11296: Standard test methods for determining average grain size. West Conshohocken: ASTM; 1996.

[14] American Standard for Testing Materals - ASTM. ASTM E56202: Standard test method for determining volume fraction by systematic manual point count. West Conshohocken: ASTM; 2002.

[15] American Standard for Testing Materals - ASTM. ASTM E8-04: Standard test methods for tension testing of metallic materials. West Conshohocken: ASTM; 2004.

[16] American Standard for Testing Materals - ASTM. ASTM E23-02: Standard test methods for notched bar impact testing of metallic materials. West Conshohocken: ASTM; 2002.

[17] Yue X., Lippold JC, Alexandrov BT, Babu SS. Continuous cooling transformation behavior in the CGHAZ of naval steels. Welding Journal. 2012;88(3):67s-75s.

[18] Bajic N., Sijacki-Zeravcic V, Bobic B, Cikara D, Arsic M. Filler metal influence on weld metal structure of microalloyed steel. Welding Journal. 2011;90(3):55s-62s.

[19] Zhang Y, Zhang H, Li J, Liu W. Effect of heat input on microstructure and toughness of coarse grain heat affected zone in $\mathrm{Nb}$ microalloyed HSLA steels. Journal of Iron and Steel Research International. 2009;16(5):73-80. http://dx.doi.org/10.1016/ S1006-706X(10)60014-3.

[20] Laitinen R. Improvement of weld haz toughness at low heat input by controlling the distribution of $\mathrm{M}$-A constituents. Oulu: Faculty of Technology, Department of Mechanical Engineering, University of Oulu; 2006.

[21] Amer E, Koo MY, Lee KH, Kim SH, Hong SH. Effect of welding heat input on microstructure and mechanical properties of simulated $\mathrm{HAZ}$ in $\mathrm{Cu}$ containing microalloyed steel. Journal of Materials Science. 2010;45(5):1248-1254. http://dx.doi. org/10.1007/s10853-009-4074-7.

[22] Mohandas T, Madhusudan Reddy G, Satish Kumar B. Heataffected softening in high-strength low alloy steels. Journal of Materials Processing Technology. 1999;88(1-3):284-294. http://dx.doi.org/10.1016/S0924-0136(98)00404-X.

[23] Kim J., Oha WI, Hwanga IS, Kimb DJ, Kimb JT. Fracture behavior of heat-affected zone in low alloy steels. Journal of nuclear materials. 2001;299(2):132-139,.

[24] Xu W, Wang QF, Pan T, Su H, Yang CF. Effect of welding heat input on simulated HAZ microstructure and toughness of a v-n microalloyed steel; Journal of Iron and Steel Research, International, 2007;14(5): 234-239. 